\title{
Correction: Event-related brain response to visual cues in individuals with Internet gaming disorder: relevance to attentional bias and decision-making
}

Bo-Mi Kim, Jiyoon Lee, A. Ruem Choi, Sun Ju Chung, Minkyung Park@, Ja Wook Koo, Ung Gu Kang (D) and Jung-Seok Choi iD

Correction to:

https://doi.org/10.1038/s41398-021-01375-x published online 5 May 2021

The original version of this article unfortunately contained a mistake. There was an error in the reference order in the discussion section. The original article has been corrected accordingly.

Published online: 26 May 2021 